\title{
Turán's graph theorem, measures and probability theory
}

\author{
Gyula O.H. Katona* \\ Rényi Institute, Hungarian Academy of Sciences \\ Budapest Pf 127, 1364 Hungary \\ ohkatona@renyi.hu
}

\section{Introduction}

Let us start with the well-known graph theorem of Turán that determines the largest number of edges in a graph on $n$ vertices containing no complete graph on $k+1$ vertices.

If $1 \leq k \leq n$ are integers, define the graph $T(n, k)$ whose vertex set is $V_{1} \cup V_{2} \cup \ldots \cup V_{k}$ where the $V_{i}$ s are pairwise disjoint and their sizes are either $\left\lfloor\frac{n}{k}\right\rfloor$ or $\left\lceil\frac{n}{k}\right\rceil$ and two vertices are adjacent if and only if they are in two different $V_{i}$ s. It is easy to see that $T(n, k)$ contains no complete graph on $k+1$ vertices. The number of edges of $T(n, k)$ is denoted by $t(n, k)$.

Theorem 1 ( [21], case $k=2$ : [14]) Let $G=(V, E)$ be a simple graph on $n=|V|$ vertices that contains no complete graph on $k+1$ vertices. Then $|E| \leq t(n, k)$.

Now we will show an application of this theorem in probability theory. The case $k=2$ will be applied in a complementary form, forbidding empty triangles and minimizing the number of edges.

*The work of the author was supported by the Hungarian National Foundation for Scientific Research, grant number NK78439. 
Corollary 1 Let $G=(V, E)$ be a simple graph with $n=|V|$, in which there is at least one edge among any 3 vertices then

$$
|E| \geq\left\lfloor\frac{(n-1)^{2}}{4}\right\rfloor
$$

(= the number of edges in the vertex-disjoint union of the complete graphs on $\left\lceil\frac{n}{2}\right\rceil$ and $\left\lfloor\frac{n}{2}\right\rfloor$ vertices, respectively).

Let $\xi$ and $\eta$ be two independent, identically distributed random vectors.

Theorem $2[6]$

$$
\operatorname{Pr}(|\xi+\eta| \geq x) \geq \frac{\operatorname{Pr}^{2}(|\xi| \geq x)}{2} .
$$

The statement of the theorem is too week if the random vectors are onedimensional, therefore we better suppose that they have at least two dimensions.

A simple geometric lemma will be needed in the proof of the theorem, that is not proved here.

Lemma 1 If $v_{1}, v_{2}, v_{3}$ are vectors in a Hilbert space, $x \leq\left|v_{1}\right|,\left|v_{2}\right|,\left|v_{3}\right|$ then there is a pair $i \neq j$ such that $x \leq\left|v_{i}+v_{j}\right|$.

Proof of Theorem 2 for the discrete case. Suppose that $v_{1}, v_{2}, \ldots, v_{m}$ are vectors and

$$
\operatorname{Pr}\left(\xi=v_{i}\right)=\frac{1}{m} \text { for } i=1,2, \ldots, m .
$$

Order them in the following way.

$$
\left|v_{1}\right| \geq\left|v_{2}\right| \geq \ldots \geq\left|v_{n}\right| \geq x>\left|v_{n+1}\right| \geq \ldots \geq\left|v_{m}\right| .
$$

The left hand side of (1) can be expressed as a ratio:

$$
\operatorname{Pr}(|\xi+\eta| \geq x)=\frac{\text { number of pairs } v_{i}, v_{j} \text { such that }\left|v_{i}+v_{j}\right| \geq x}{m^{2}}
$$

Separating the cases $i \neq j$ and $i=j$ and observing that $\left|v_{i}+v_{i}\right|>x$ when $1 \leq i \leq n$ the following lower estimate is obtained for (2).

$$
\frac{2 \cdot\left(\text { number of pairs } v_{i}, v_{j} \text { such that }\left|v_{i}+v_{j}\right| \geq x(i<j)\right)+n}{m^{2}} .
$$


Introduce the graph $G(V, E)$ where $V=\left\{v_{1}, v_{2}, \ldots, v_{n}\right\}$ and $\left\{v_{i}, v_{j}\right\} \in E$ iff $\left|v_{i}+v_{j}\right| \geq x(i \neq j)$. Then (3) can be written as

$$
\frac{2|E|+n}{m^{2}}
$$

By Lemma $1 G$ contains no empty triangle, and Corollary 1 implies that

$$
\frac{2\left\lfloor\frac{(n-1)^{2}}{4}\right\rfloor+n}{m^{2}} \geq \frac{2 \frac{n^{2}-2 n}{4}+n}{m^{2}}=\frac{\frac{n^{2}-2 n}{2}+n}{m^{2}}=\frac{1}{2}\left(\frac{n}{m}\right)^{2}
$$

is a lower estimate on (4) (and consequently on (2)). However, $\frac{n}{m}$ is nothing else but $\operatorname{Pr}(|\xi| \geq x)$, proving Theorem 2 for the discrete case.

The most natural way to prove it for the general (continuous) case is to approximate the distribution with a discrete one. This was done in [6] in a clumsy way: an unnecessary additional condition was supposed on the distribution of the random vector. This approach was properly completed by Sidorenko in [16].

Another, prettier way is to generalize Turán's theorem in a "continuous" way, where the underlying set is a measure space and the the measure replaces the "number of" vertices/edges. This will be introduced in Section 2. We will also show how to find the "continuous version" of an extremal result on graphs and hypergraphs.

Section 3 gives a sample of further inequalities for the probability distributions of random vectors, while Section 4 shows the connections to Szemerédi's Regularity Lemma.

\section{Continuous versions of results in extremal graph theory}

Consider the interval $[0,1]$ as a set of vertices of an infinite graph. Then a directed edge of the graph is a pair $(a, b)(0 \leq a, b \leq 1)$. This can also be considered as a point of the unit square. Therefore a directed graph will be a subset $E$ of the unit square. This is typically an infinite set, therefore we cannot really speak about the number of edges, instead we will consider its measure $\mu(E)$. The analogue of a simple graph now will be a symmetric, measurable set $E \subset[0,1]^{2}$. 
Now find the analogue of the condition in Corollary 1. Choose 3 distinct vertices $0 \leq a<b<c \leq 1$ in the interval $[0,1]$. According to our "finite" condition

$$
\text { at least one of the pairs }(a, b),(a, c),(b, c) \text { must be in } E \text {. }
$$

Looking at it geometrically, the 3 distinct points $a, b, c$ as coordinates determine 9 crossing points in the unit square. 3 of them are loops they are on the diagonal. At least one of the other 6 points must be in $E$. Because of the symmetry it means only 3 points. Does this condition imply that the measure of $E$ is at least $\frac{1}{2}$ ? Define

$$
W=\left\{(x, y): 0 \leq x, y \leq \frac{1}{2}\right\} \cup\left\{(x, y): \frac{1}{2}<x, y \leq 1\right\}-\left\{\left(\frac{1}{2}, \frac{1}{2}\right)\right\} .
$$

It is easy to see that $W$ satisfies (i) and $\mu^{2}(W)=\frac{1}{2}$ if $\mu^{2}$ is the area. We will show now another measure where this is not true. Let the measure on $[0,1]$ be defined as $\mu_{1}\left(\left\{\frac{1}{2}\right\}\right)=\frac{1}{2}$ and $\mu_{1}(A)=\left(\frac{1}{2} \cdot\right.$ (the "length" of $A$ )) if $\frac{1}{2} \notin A$. Its "square" $\mu_{1}^{2}$ gives the value $\mu_{1}^{2}(W)=\frac{3}{8}$. The obvious cause of this counter-example is that there is an element with positive measure. In general a measure $\mu$ is atomless if for any measurable $A$ with positive measure there is a measurable $B \subset A$ satisfying $0<\mu(B)<\mu(A)$. Now we can formulate the following theorem.

Theorem 3 (See [1], [7], [11], [12].) Suppose that E is a symmetric subset of $[0,1]^{2}$ satisfying (i). If $\mu$ is an atomless measure on $[0,1]$ then

$$
\frac{1}{2} \leq \mu^{2}(E)
$$

holds for the product measure $\mu^{2}$.

However we need Theorem 2 also for distributions with "atoms" that is with single elements with positive probability. The discrete case had this property. Observe that the reason why our proof was working in the discrete case, was that we could add the term $n$ in (3), since $|v+v|>x$ holds when $v \geq x(>0)$. In other words in our application all the loops were in the graph. This fact shows the form that is really needed.

Theorem 4 (See [7], [11], [12].) Suppose that $E$ is a symmetric subset of $[0,1]^{2}$ satisfying (i) and containing the diagonal. Then

$$
\frac{1}{2} \leq \mu^{2}(E)
$$

holds for any product measure $\mu^{2}$ (see below). 
In order to be able to prove Theorem 2 in full generality this statement has to be stated for arbitrary probability spaces rather than the interval $[0,1]$. Let $M=(X, \sigma, \mu)$ be a probability space, where $X$ is the set of elementary events, $\sigma$ is a sigma algebra on $X$ and $\mu$ is a finite measure on $\sigma$. Then the direct product of $M$ with itself is $M^{2}=\left(X^{2}, \sigma^{2}, \mu^{2}\right)$ where $\sigma_{2}$ is induced by the products of the members of $\sigma$ and $\mu^{2}$ is the product measure.

Theorem 5 (See [7], [11], [12].) Suppose that $E$ is a symmetric subset of $X^{2}$ satisfying (i) and containing all elements of form $(y, y)(y \in X)$. Then

$$
\frac{1}{2} \mu^{2}(X) \leq \mu^{2}(E)
$$

Now we are ready to prove Theorem 2 in full generality.

Proof of Theorem 2. Let $X=\{v:|v| \geq x\}, \sigma$ is the restriction of the original sigma algebra for $X, \mu(A)=\operatorname{Pr}(A)$. Define the graph $(X, E)$ by $\left(v_{1}, v_{2}\right) \in E$ if and only if $v_{1}, v_{2} \in X,\left|v_{1}+v_{2}\right| \geq x$ holds. Lemma 1 ensures that $E$ satisfies (i). $(v, v) \in E$ is trivial for $v \in X$. Theorem 5 can be applied:

$$
\begin{gathered}
\operatorname{Pr}(|\xi+\eta| \geq x) \geq \operatorname{Pr}(|\xi+\eta| \geq x,|\xi|,|\eta| \geq x)= \\
\mu^{2}(E) \geq \frac{1}{2} \operatorname{Pr}^{2}(|\xi| \geq x) .
\end{gathered}
$$

Observe that the class of all finite graphs containing no empty triangle has the following property. Taking an induced subgraph of a member of this class also belongs to the class. This property is generalized in the following way. A class $\mathcal{G}$ of finite graphs is called induced hereditary if every induced subgraph $G^{\prime}$ of $G \in \mathcal{G}$ is also in $\mathcal{G}$. The extremal graph problem in this generality can be formulated as follows.

$$
H(n, \mathcal{G})=\min \frac{|E|}{n^{2}}
$$

where the minimum is taken for all edge sets $E$ of graphs in $\mathcal{G}$ with $n$ vertices.

The continuous analogue is very similar. A class $\mathcal{G}$ of graphs $G=(X, E)$ given on the measure space $(X, \sigma, \mu)$ is called induced hereditary if every subgraph of a member of $\mathcal{G}$, induced by a measurable vertex set, is also in $\mathcal{G}$. The asymptotic optimum here is

$$
H(M, \mathcal{G})=\inf \frac{\mu^{2}(E)}{\mu(X)^{2}}
$$


where $E$ is the edge set of a member of $\mathcal{G}$. Suppose that the finite induced subgraphs of the members of the infinite $\mathcal{G}$ also belong to $\mathcal{G}$. Then we can use the same notation for the finite and infinite case.

Theorem 6 ([1], [7]) $\mathcal{G}$ is an induced hereditary class of graphs on the atomless measure space $M$ then

$$
H(M, \mathcal{G}) \geq H(n, \mathcal{G})
$$

holds.

One can see that the condition on atoms cannot simply be deleted without the usage of loops. We will see that there is a condition that is weaker in some cases than just adding all loops. Let $u$ be a vertex of the graph $G$. The graph $G^{u}$ is consists of the same vertex set as $G$, but $u$ is replaced by two new vertices, $u^{\prime}$ and $u^{\prime \prime}$ where these new vertices are adjacent to other vertices if and only if $u$ was. If there is no loop at $u$ then $u^{\prime}, u^{\prime \prime}$ is not an edge in $G^{u}$, otherwise $u^{\prime}, u^{\prime \prime}$ is an edge with loops at both ends. We say that the class $\mathcal{G}$ is doublable if $G \in \mathcal{G}$ implies $G^{u} \in \mathcal{G}$ for every vertex $u$ of $G$.

Theorem 7 [7] Let $\mathcal{G}$ be an induced hereditary, doublable class of graphs, where loops are allowed, on a measure space $M$. Then

$$
H(M, \mathcal{G}) \geq H(n, \mathcal{G})
$$

holds.

We will now formulate the "continuous" version of the Erdős-StoneSimonovits theorem, a strong generalization of our Theorem 5. Recall the finite form. (We apologise for taking the unusual complementing setting, this is caused by the applications where this complementing form is more convenient.) Let $F$ be a fixed finite simple graph. Then $\mathcal{G}(F)$ denotes the set of graphs (with possible loops) not containing an empty copy of $F$. $\chi(F)$ is the chromatic number of $F$.

Theorem 8 [3] [19] [2]

$$
H(n, \mathcal{G}(F))=\frac{1}{\chi(F)-1} .
$$

Now Theorems 6 and 7 imply the continuous version. 
Theorem 9 If either $M$ is atomless or $\mathcal{G}(F)$ is doublable then

$$
H(M, \mathcal{G}(F))=\frac{1}{\chi(F)-1} .
$$

(Theorems 6 and 7 only imply the inequality $\geq$. The equality is a consequence of the easy construction consisting of $\frac{1}{\chi(F)-1}$ squares of size $1 /\left(\frac{1}{\chi(F)-1}\right)$.)

All of the definitions and statements of this section can be treated in a more general context. A directed $r$-graph is a pair $(X, E)$ where $E$ consists of sequences $\left(x_{1}, x_{2}, \ldots, x_{r}\right)\left(x_{i} \in X\right)$. Then we have "loops" of different sizes depending on the repetitions among the $x_{i}$ s. The main difficulty in the application of these is that there are relatively few extremal results on hypergraphs in the finite case. But the transfer from finite to the infinity works like in the case of ordinary graphs. See [7].

Let us have one more comment on the concept of doubling. If a(n ordinary) graph has a vertex without loop, then two doublings form an empty triangle at this vertex. Therefore if $\mathcal{G}$ is a class of graphs without empty triangles and it is doublable then all members must have loops at every vertex. The effect of doubling is trivial here. We will show an example after Lemma 4 when this is not the case.

[8] is treating the continuous versions of some other types of extremal problems, when the minimum of the transformed form of a structure is sought. A typical example is the Shadow theorem ([13],[5]).

\section{Inequalities on the distribution of the length of two random vectors}

Theorem 2 gave a lower estimate on $\operatorname{Pr}(|\xi+\eta| \geq x)$ using the distribution function of the length of one vector at the same place, $x$. It is worth mentioning that this estimate is sharp, if the dimension is at least 2 , in the sense that for any given $p$ there is a distribution of random vectors where $\operatorname{Pr}(|\xi| \geq x)=p$ and $\operatorname{Pr}(|\xi+\eta| \geq x)=\frac{1}{2} p^{2}$. However there is a sharper estimate in Feller's book [4] for the case of one dimension.

A natural question is what estimate one can give on $\operatorname{Pr}(|\xi+\eta| \geq x)$ using the distribution function $\operatorname{Pr}(|\xi| \geq x)$ at another place, say at $c x$ where $c$ is a positive constant. More precisely, try to give the best function $f$ such that

$$
\operatorname{Pr}(|\xi+\eta| \geq x) \geq f(\operatorname{Pr}(|\xi| \geq c x)) .
$$


"Best" means here that there is a random vector $\xi$ for every $0 \leq p \leq 1$ such that $p=\operatorname{Pr}(|\xi| \geq c x)$ and $\operatorname{Pr}(|\xi+\eta| \geq x)=f(p)$ or at least there is a sequence of $\xi$ s for every $0 \leq p \leq 1$ such that $p=\operatorname{Pr}(|\xi| \geq c x)$ and $\operatorname{Pr}(|\xi+\eta| \geq x) \rightarrow f(p)$.

Theorem 10 [9] Let $H$ be an infinite-dimensional Hilbert space and $\xi, \eta$ independent, identically distributed random variables in $H$, then the best $f$ are the following ones.

$$
\begin{aligned}
& f(p)=\left\{\begin{array}{ll}
\frac{1}{2} & \text { if } p \geq \frac{1}{3}, \\
2 p-\frac{3}{2} p^{2} & \text { otherwise, }
\end{array} \quad \text { when } \frac{5}{2} \leq c<\infty ;\right. \\
& f(p)=\left\{\begin{array}{ll}
\frac{1}{2} & \text { if } p \geq \frac{1}{2}, \\
2 p(1-p) & \text { otherwise, }
\end{array} \text { when } \frac{3}{2} \leq c<\frac{5}{2} ;\right. \\
& f(p)=\left\{\begin{array}{ll}
-\frac{1}{2}+2 p-p^{2} & \text { if } p \geq \frac{1}{2}, \\
p^{2} & \text { otherwise, }
\end{array} \quad \text { when } \frac{\sqrt{5}}{2} \leq c<\frac{3}{2} ;\right. \\
& f(p)=\frac{1}{2} p^{2} \quad \text { when } 1 \leq c<\frac{\sqrt{5}}{2} ; \\
& f(p)=\frac{1}{k-1} p^{2} \text { when } \sqrt{\frac{k-1}{2(k-2)}} \leq c<\sqrt{\frac{k-2}{2(k-3)}}(4 \leq k<\infty) ; \\
& f(p)=0 \text { when } 0 \leq c \leq \frac{1}{\sqrt{2}} \text {. }
\end{aligned}
$$

Let us give some hints concerning the proof in some cases. The proof of the 5 th row is based on the following lemma.

Lemma 2 If $a_{1}, a_{2}, \ldots, a_{k}$ are vectors in a Hilbert space, $\left|a_{i}\right| \geq 1(1 \leq i \leq k)$ then there is a pair $i \neq j$ with

$$
\left|a_{i}+a_{j}\right| \geq \sqrt{\frac{k-1}{2(k-2)}} .
$$

Then the "continuous" version (plug in Theorem 1 into Theorem 7) can be used for the infinite graph where the vertices are the vectors of length $\geq c x$, the edges are defined by $\left\|a_{i}+a_{j}\right\| \geq x$.

The proof of the 3rd row needs some small new ideas. The following geometrical lemma is applied. 
Lemma 3 If $a_{1}, a_{2}, a_{3}$ are vectors in a Hilbert space, $\left|a_{1}\right|,\left|a_{2}\right| \geq \frac{\sqrt{5}}{2}$ then there is a pair $i \neq j$ with

$$
\left|a_{i}+a_{j}\right| \geq 1
$$

The novelty here is that we cannot disregard the short vectors. This is why the extremal graph statement must have two kinds of vertices. $V_{2}$ is the set of vectors with length at least $\frac{\sqrt{5}}{2}$, while $V_{1}$ is the set of small vectors, and a "two-part" Turán type theorem should be used.

Lemma 4 Let the vertex set of the graph $G=(V, E)$ divided into two parts: $V=V_{1} \cup V_{2}, V_{1} \cap V_{2}=\emptyset,\left|V_{1}\right|=n_{1},\left|V_{2}\right|=n_{2}, n_{1}+n_{2}=n$. If the graph contains no empty triangle with at least two vertices from $V_{2}$ then

$$
|E| \geq\left\{\begin{array}{cl}
\left\lfloor\frac{2 n_{1} n_{2}-n_{1}^{2}+n_{2}^{2}-2 n_{2}+1}{4}\right\rfloor & \text { if } n_{1} \leq n_{2} \\
\left(\begin{array}{c}
n_{2} \\
2
\end{array}\right) & \text { if } n_{1} \geq n_{2} .
\end{array}\right.
$$

Of course here we need a generalization of the transfer from the finite to infinite for the case of two-part extremal theorems

In this case the property that the class of graphs is doublable does not imply that all loops have to be present. The class of graphs containing no empty triangle with at with at least two vertices from $V_{2}$, having all loops in $V_{2}$, but none in $V_{1}$ is obviously doublable.

Why did we consider the case of an infinite-dimensional Hilbert space? Because a space with finite dimension causes restrictions on the constructions. The geometric problems needed for the solutions are mostly unsolved even in an asymptotic way. See [9].

Another generalization is given in [16]. It gives the best lower estimate for $\operatorname{Pr}(|a \xi+b \eta| \geq x)$ using the distribution function at a 3rd place: $\operatorname{Pr}(|\xi| \geq c x)$.

An interesting variant of the problem of Theorem 8 is when it is supposed that the distribution of the random vector is symmetric. The solution is due to Sidorenko [17]. The interested reader might also study the paper [18].

There are some modest results on the sum of 3 random vectors in [10] (see for some remarks also [12]), where an attempt to give a good lower estimate on $\operatorname{Pr}\left(\left|\xi_{1}+\xi_{2}+\xi_{3}\right| \geq x\right)$ can be found. The largest difficulty is that the necessary finite extremal problems for 3-graphs are unsolved.

But there are many other unsolved problems here. 
Open problem 1 Find the "best" two-variable function $f(u, v)$ such that

$$
\operatorname{Pr}(|\xi+\eta| \geq x) \geq f(\operatorname{Pr}(|\xi| \geq y), \operatorname{Pr}(|\xi| \geq z))
$$

holds and it is sharp.

Open problem 2 Find "good" functionals F such that

$$
\operatorname{Pr}(|\xi+\eta| \geq x) \geq F(\text { distribution function of }|\xi|)
$$

holds.

\section{The Szemerédi connection}

The main goal of Section 2 was to survey some results on "continuous" versions of extremal graph theorems. Szemerédi's Regularity Lemma [20] is of very different nature, it claims that every large graph has a certain structure. Yet, there is some similarity. The same attitude can be applied to both of them: looking at a continuous variant.

Let us start with a very heuristic form of the Regularity Lemma. It claims that, given an integer $Z$ and a real $\varepsilon>0$, if the number $N$ of vertices of a graph is large enough $(N>N(Z, \varepsilon))$ then its vertex set can be divided into nearly equally sized subsets $C_{1}, C_{2}, \ldots, C_{K}$ where ||$C_{i}|-| C_{j}|| \leq 1$ and $Z \leq K \leq Z+M(Z, \varepsilon)$ in such a way that the edges between almost (depends on $\varepsilon$ ) every pair $\left(C_{i}, C_{j}\right)$ behave like a random graph. (Here the measure of randomness also depends on $\varepsilon$.) These pairs of subsets $C$ are called called regular pairs. Assume here that $K$ divides $N$ and use the notation $\frac{N}{K}=n$.

Suppose that the $N$ vertices of the graph are ordered in such a way, that the elements of $C_{1}$ are the first ones, then the elements of $C_{2}$ come, and so on. Divide the unite square $[0,1]^{2}$ into $N \times N$ small squares of equal sizes. Define a function $g$ on $[0,1]^{2}$ giving the constant value 1 on the small square determined by the $i$ th row and $j$ th column if $\{i, j\}$ is an edge and 0 otherwise. Suppose that $\left(C_{k}, C_{\ell}\right)$ is a regular pair. It corresponds to a square $S_{k, \ell}$ of size $n \times n$. Let $p_{k, \ell}$ denote the ratio (number of values 1$) / n^{2}$ in this square. Make more precise what we understand under the random behaviour: choosing any rectangle with non-negligible sides $u$ and $v$, the ratio (number of values 1 in this rectangle) $/ u v$ is very close to $p_{k, \ell}$. One can heuristically say that the values 1 are uniformly distributed in $S_{k, \ell}$ with density $p_{k, \ell}$. Replace the 0,1 
function by a constant function $p_{k, \ell}$ in $S_{k, \ell}$, since $n$ is large and the local irregularities are negligible. On the other hand $K$ is also large, therefore the number of irregular pairs, where $p_{k, \ell}$ is undefined, is negligible, again. In this way we replaced the picture of the graph with a function being constant in the squares $S_{k, \ell}$.

The precise description of this heuristic statement can be read in a paper of L. Lovász and B. Szegedy [15].

\section{Acknowledgement}

The author is indebted to Miki Simonovits for his valuable suggestions and help.

\section{References}

[1] B. Bollobás, Measure graphs, J. London Math. Soc.(1980) 401-407.

[2] P. Erdős, M. Simonovits, A limit theorem in graph theory, Studia Sci. Math. Hungar. 1(1966) 51-57.

[3] P. Erdős, A.H. Stone, On the structure of linear graphs, Bull. Amer Math. Soc. 52(1946) 1087-1091.

[4] W. Feller, An introduction to probability theory and its applications, II Wiley-Chapman, page 147.

[5] G. Katona, A theorem on finte sets, Theory of Graphs, Proc. Coll. held at Tihany, 1966, Akadémiai Kiadó, pp. 187-207.

[6] Gyula Katona, Graphs, vectors, probabilistic inequalities, Matematikai Lapok 20(1969) 123-127 (in Hungarian).

[7] G.O.H. Katona, Continuous versions of some extremal hypergraph problems, in: Coll. Math. Soc. Bolyai 18(1976) 653-678.

[8] G.O.H. Katona, Continuous versions of some extremal hypergraph problems, II, Acta Math. Acad, Sci. Hungar. 35(1980) 67-77.

[9] G.O.H. Katona, "Best" estimations on the distribution of length of sums of two random vectors, Z. Wahrsch. Vew. Gebiete 60(1982) 411-423. 
[10] G.O.H. Katona, Sums of vectors and Turán's problem for 3-graphs, European J. of Combinatorics 2(1981) 145-154.

[11] G.O.H. Katona, Continuous versions of extremal combinatorial theorems with application in probability theory, Academic Doctor Thesis, Hungarian Academy of Sciences, Budapest, 1981 (in Hungarian).

[12] G.O.H. Katona, Probabilistic inequalities from extremal graph results (a survey), Annals of Discrete Mathematics 28(1985) 159-170.

[13] J.B. Kruskal, The number of simplices in a complex, Mathematical Optimization Techniques, University of Calif. Press, (Berkeley and Los Angeles, 1963), pp. 251-278.

[14] W. Mantel, Problem 28, Wiskunde Opgaven 10(1907) 60-61.

[15] László Lovász; Balázs Szegedy, Szemerédi's Lemma for the analyst, Geom. Funct. Anal. 17(2007) 252-270.

[16] A.F. Sidorenko, Extremal estimates of probability measures and their combinatorial nature, Izv. Acad. Nauk. SSSR 46(1982) 535-568 (in Russian).

[17] A.F. Sidorenko, A Precise Inequlity for the Sum of Two Random Vectors with Symmetric Distribution, Theory Probab. Appl. 35(1990) 613-617.

[18] A.F. Sidorenko, Inequalities in probability theory and turán-type problems for graphs with colored vertices, Random Structures and Algorothms 2(1991) 73-99.

[19] M. Simonovits, A method solving extremal problems in graph theory, in: Theory of Graphs, Proc. Coll. Tihany, 1966, Akadémiai Kiadó, 1968, pp. 279-319.

[20] E. Szemerédi, Regular partitions of graphs, in: Problèmes Coimbatoires et Théorie des Graphes (Colloq. Internat. CNRS, Univ. Orsay, Orsay, 1976), Colloq. Internat. CNRS 260 CNRS, Paris, 1978, pp. 399-401.

[21] P. Turán, On a problem in graph theory (in Hungarian) Mat. Fiz. Lapok 48(1941) 436-452. 